\title{
Mixture Interactions at Mammalian Olfactory Receptors are Dependent on the Cellular Environment
}

\section{Elizabeth Corey}

University of Florida

\section{Sergei Zolotukhin}

University of Florida

\section{Barry Ache}

University of Florida

Kirill Ukhanov ( $D$ kirill.ukhanov@ufl.edu )

University of Florida

\section{Research Article}

Keywords: olfactory receptors (ORs), Olfr73, vivo, vitro

Posted Date: December 23rd, 2020

DOI: https://doi.org/10.21203/rs.3.rs-123541/v1

License: (9) This work is licensed under a Creative Commons Attribution 4.0 International License.

Read Full License

Version of Record: A version of this preprint was published at Scientific Reports on April 29th, 2021. See the published version at https://doi.org/10.1038/s41598-021-88601-0. 


\section{Abstract}

Functional characterization of mammalian olfactory receptors (ORs) remains a major challenge to ultimately understanding the olfactory code. Here, we compare the responses of the mouse Olfr73 ectopically expressed in olfactory sensory neurons using AAV gene delivery in vivo and expressed in vitro in cell culture. The response dynamics and concentration-dependence of agonists for the ectopically expressed Olfr73 were similar to those reported for the endogenous Olfr73, but the receptor failed to mediate the antagonism between its cognate agonist and several antagonists reported for the OR expressed in vitro. Expressing the $\mathrm{OR}$ in vitro under the same conditions of stimulation retained the reported antagonism, but it was dependent on the duration of stimulation. Our findings suggest that both the cellular environment and the stimulus dynamics shape the functionality of Olfr73 and argue that characterizing ORs in 'native' conditions provides the most complete understanding of ligand-OR interactions.

\section{Introduction}

Mammalian olfactory receptors (ORs) form the largest gene family of all G-protein coupled receptors (GPCRs), with approximately 1,100 members in rodents and 400 in humans ${ }^{1}$. Such a large number of input channels raises the challenge to understand the logic of olfactory coding. This fundamental question has been the renewed focus of number of recent studies that have added to our understanding by showing that ORs encode information through broad-based excitation and inhibition [e.g. ${ }^{2-4}$ ]. This knowledge in turn adds weight to the importance of identifying the full response spectrum, i.e., the molecular receptive range (MRR), of individual ORs that includes both the excitatory and inhibitory ligands for a given $\mathrm{OR}$.

ORs have proven difficult to deorphanize by expressing them in non-olfactory cells, in large part due to the poor surface expression of recombinant ORs in cultured cells. Several chaperon proteins, including small GTPases RTP1s, Ric8B and REEP1, control trafficking of the OR protein from trans-Golgi to the plasma membrane ${ }^{5}$. Moreover, ORs are quite diverse and lack an identifiable consensus motif for membrane and olfactory cilia trafficking ${ }^{6}$. Some progress has been made by fusing a short lead sequence of the first 20 amino acids from bovine rhodopsin (Rho-tag) in-frame to the N-terminus of an OR to increase surface expression in cultured cells ${ }^{5,7}$. Two assays were also developed to functionally characterize ORs utilizing the non-native promiscuous Ga15 protein coupled to phospholipase-C dependent calcium release to promote signaling or an assay based on the interaction of the $\mathrm{OR}$ co-expressed with $\mathrm{G}_{\text {olf }}$ in measuring odorant evoked production of CAMP ${ }^{7}$. The latter approach was further developed to allow highthroughput analysis utilizing the cAMP-dependent upregulation of a Cre response element fused to SEAP phosphatase or luciferase as a readout ${ }^{8,9}$. This assay requires long incubation with the ligand of interest, up to several hours, which is unlikely to occur in vivo where odorant availability is limited by the rapid dynamics of the sniff cycle ${ }^{10}$. 
These constraints have been partially alleviated by characterizing ORs in olfactory sensory neurons (OSNs) in vivo, e.g., using transgenic animals expressing a fluorescent protein or a reporter under the promoter of a given $\mathrm{OR}^{11}$. Several ORs of different classes have been successfully characterized using this approach ${ }^{11,12}$. A less time-consuming approach has been to ectopically express a known OR through virally assisted gene delivery to native OSNs. The rodent Olfr41 receptor (17, MOR103-15) was the first to be successfully characterized by adenoviral gene delivery ${ }^{13}$, and since, other recombinant viral vectors, including retrovirus and Herpes simplex, have been used to ectopically express ORs and vomeronasal receptors in their respective sensory neurons ${ }^{13,14}$.

Here, using the well-characterized mouse OR, Olfr73 ${ }^{15-17}$, and recombinant adeno-associated virus (rAAV) vector-mediated gene delivery, we show markedly different sensory responses when using two contrasting assay platforms, in vitro and in vivo. When ectopically expressed in vivo, Olfr73 displayed the predicted responses to their cognate ligands, while the same $\mathrm{OR}$, heterologously expressed in vitro and challenged with prolonged application of two odorants, produced, surprisingly, an additive agonism to the otherwise antagonist odorant and not the predicted antagonism or lack of a response. These findings suggest that the functionality driven by Olfr73, and likely other ORs, is dependent on the innate cellular environment and stimulus dynamics, underscoring the need to characterize ORs in 'native' conditions in order to provide the most complete understanding of ligand-OR interactions.

\section{Results}

\section{rAAV2/5 as a vector for gene delivery to mammalian OSNs}

Given limited prior knowledge of the tropism of different AAV serotypes in the mammalian nasal epithelium, we tested an rAAV5-based vector not reported previously to specifically transduce rodent OSNs but known to display a broad tissue tropism ${ }^{18}$. A bicistronic cassette encoding firefly Luciferase and $m A p p l e$ fluorescent protein linked by the furin-cleavage signal and ribosome skipping peptide $2 \mathrm{~A}$ from a foot-and-mouth virus (Fig. 1A, top), was used to produce the rAAV5 vector ${ }^{19}$. A single dose of rAAV5 (10-20 $\mu$ l, titer ca. $\left.10^{11} \mathrm{vg} / \mathrm{ml}\right)$ was administered intranasally to anesthetized rats and mice. Robust Luciferase activity, restricted to the nasal cavity only, was detected 7 days later in a mouse (Figure 1A). The same animal used for in vivo Luciferase imaging was subsequently transcardially perfused and analyzed for the mApple expression in the olfactory epithelium (OE) (Figure 1B). rAAV5 successfully induced expression in multiple OSNs along with non-sensory sustentacular cells (Figure 1B). Similarly, en face imaging of the acutely isolated $\mathrm{OE}$ from the mouse treated with the rAAV 5 revealed a mixed population of transduced OSNs and sustentacular cells (Figure 1C). At higher magnification multiple cilia emanating from the dendritic knob of the OSN could be clearly resolved (Figure 1D).

\section{rAAV5-mediated transduction of the olfactory epithelium and ectopic expression of the OR in rodent OSNs}


Following initial characterization of transduction of the OE by rAAV5, we subcloned full-length Olfr73, a Class II OR (other names MOR174-1, mOR-EG) ${ }^{1}$ and a genetically-encoded calcium indicator GCaMP3 in to the same vector by substituting the respective ORFs (Figure 2A, top). We hypothesized that OSNs should have all necessary endogenous cellular mechanisms for the correct expression and trafficking of the OR protein to cilia. Hence, no additional modification of the OR gene via fusing the N-terminus tag to improve plasma membrane localization was made, thus ensuring that the expression in OSNs identical to its natural counterparts.

First, functional expression of the OR and GCaMP3 was validated in HEK293T cells by co-transfecting the pTR plasmid encoding the bicistronic CDNA used to produce rAAV5 with the promiscuous G-protein Ga15, conferring coupling to the downstream calcium release signaling cascade. A $5 \mathrm{sec}$ application of eugenol $(100 \mu \mathrm{M})$ evoked robust elevation of intracellular calcium (Supplemental Figure S6B). Our result confirmed earlier reports of functional expression of untagged full-length Olfr73 in HEK293 cells ${ }^{20}$ and proved the functionality of the bicistronic expression cassette.

Next, a single intranasal dose of rAAV5 was used to induce expression of the bicistronic construct in the $\mathrm{OE}$, as evidenced by the fluorescence of GCaMP3 in many cells at a level sufficiently strong to visualize dendritic knobs with their attached cilia (Figure 2, Supplemental Figure S1D). This allowed visualizing functional activity within the transduction compartment in semi-intact tissue with high resolution (Supplemental movie S1, S2). In response to a brief 5-sec pulse of eugenol (1 mM), we observed an odorevoked global cilia-to-knob response as well as residual activity in the cilia post-stimulation (Figure 2B, arrows). These results clearly suggest that both Olfr73 and GCaMP3 were properly localized to the transduction compartment. Transduced mouse OSNs responded to lower concentrations of eugenol (5 $\mu \mathrm{M}$ ) and showed left-shifted concentration-response functions compared to rat OSNs (Figure 2D; 3D). Unexpectedly, methylisoeugenol (MIEG, $1 \mathrm{mM}$ ), a previously characterized antagonist of eugenol on Olfr73 ${ }^{15}$, also evoked a measurable response. The latter finding set the foundation of the current study.

To confirm the general utility of the rAAV5 delivery and expression system we subcloned another mouse OR, a Class I Olfr599 (MOR23-1), using the same bicistronic cassette (Fig. 1A, top). First, we verified that Olfr599 was functionally expressed in our expression system either as a rho-tagged OR or as the untagged OR. Octanoic acid, a ligand of the $\mathrm{OR}^{8}$ consistently activated a response in a concentrationdependent manner with an $\mathrm{EC}_{50}=70 \mu \mathrm{M}(\mathrm{n}=21)$ (Supplemental Figure S1A,B). Importantly, transfection of untagged Olfr599 using the bicistronic pTR-Olfr599-furin2A-GCaMP3 plasmid also resulted in functional expression of the OR along with the calcium reporter (Supplemental Figure S1C). Similarly, intranasal infusion of rAAV2/5-Olfr599-furin2A-GCaMP3 induced expression as evident by GCaMP3 fluorescence in the knobs of mouse OSNs (Supplemental Figure S1D). Octanoic acid and octanal (each at $100 \mu \mathrm{M})$ and a mix of IBMX and forskolin $(100 / 10 \mu \mathrm{M})$, a chemical activator of most OSNs, all evoked reliable neuronal responses (Supplemental Figure S1E).

Finally, we asked whether rAAV-mediated potential over-expression of ectopic ORs could have induced an elevated basal activity known to be dependent on the type of the OR ${ }^{21}$. As a proxy for basal neuronal 
activity, we counted Olfr73-GCaMP3-OSNs (Olfr73-OSNs) showing aberrant calcium bursts. In the entire pool of Olfr73-OSNs, we found only $6.44 \%$ (13 of 202 cells) spontaneously active Olfr73-OSNs, generating bursts at $0.08 \pm 0.03 \mathrm{~Hz}(n=13)$. These values were even lower than $12.9 \%$ of unidentified OSNs bursting at $0.16 \pm 0.01 \mathrm{~Hz}$ reported in a recent study ${ }^{22}$. This confirms previously published data of low basal activity of native Olfr73-ires-tauGFP OSNs, as well as Olfr73 expressed in HEK293 cells 3,21. This validated the prior understanding that ectopic expression of a different OR in a mature OSN can be used as a reliable model to study ORs in the native cellular environment ${ }^{13}$.

\section{Pharmacological profile of OSNs ectopically expressing Olfr73}

First, we validated that the $O R$ in question confers its previously characterized molecular receptive range (MRR). Olfr73-OSNs were challenged with two ligands outside the known MRR of Olfr73, amyl acetate and acetophenone (both at $100 \mu \mathrm{M}$ ), followed by increasing concentrations of eugenol. Amyl acetate and acetophenone evoked variable responses in different Olfr73-OSNs, reflecting the expected expression of different endogenous ORs in these cells (Figure 3A), while application of eugenol consistently evoked a response in all cells in a concentration-dependent manner (Figure 3B). In each case, the response profile matched that expected of Olfr73 (Figure 3B). The agonists eugenol and isoeugenol evoked responses in mouse Olfr73-OSNs in a concentration-dependent manner, yielding $\mathrm{EC}_{50}$ values of $4.8 \pm 1.1 \mu \mathrm{M}(\mathrm{n}=18)$ and $117 \pm 45 \mu \mathrm{M}(\mathrm{n}=24)$, respectively (Figure 3B,C). So did rat Olfr73-OSNs, but they were nearly 10 -fold less sensitive to eugenol than mouse OSNs, yielding a right-shifted dose response curve with an $\mathrm{EC}_{50}$ of $41.2 \pm 12.4 \mu \mathrm{M}(\mathrm{n}=13)$ (Figure 3D). MIEG was a partial agonist for Olfr73 expressed in native mouse OSNs, yielding an $\mathrm{EC}_{50}$ of $430 \pm 130 \mu \mathrm{M}(\mathrm{n}=15)$ (Figure 3D). Isosafrole, a putative antagonist of Olfr73 ${ }^{16}$, and nootkatone, a putative highly potent agonist of 0 lfr $73^{9}$, even applied at high concentration (1 mM), only activated Olfr73-OSNs at $25 \%$ (isosafrole, $n=11$ ) and $50 \%$ (nootkatone, $n=7$ ) of the response to eugenol (1 mM) (Figure 4A, B, D). The potency of nootkatone was consistent for both rat and mouse Olfr73-OSNs (Figure 4B), suggesting that in native OSNs nootkatone acts only as a partial agonist. Lastly, we showed that the inhibitor of adenylyl cyclase, SQ22,536 (200 $\mu \mathrm{M})$, strongly reduced the response to MIEG, confirming that it was mediated through a canonical cAMP-dependent signaling pathway (Supplemental Figure S2) and that MIEG is a low-potency partial agonist for Olfr73 ectopically expressed in native OSNs.

\section{Ectopically expressed Olfr73 does not mediate ligand- dependent antagonism in native OSNs}

Interaction of ligands in binary mixture may show antagonism even though one of the components alone acts as a partial or low-potency agonist ${ }^{23,24}$ so we sought to further characterize the interaction between eugenol and several potential antagonists. First, we established the response profile of the Olfr73-OSNs 
to repeated stimulation with a 5-s pulse of eugenol $(100 \mu \mathrm{M})$ followed by the pulse of the putative antagonist (1 mM) (Figure 4A). While MIEG evoked responses of similar amplitude relative to eugenol in both rat and mouse Olfr73-OSNs (Figure 4B), we limited these experiments to mouse OSNs to exclude any issues related to the reduced sensitivity of rat Olfr73-OSNs (Figure 3D). In addition to MIEG and isosafrole, we tested the chemical dimer of isoeugenol (di-IEG, $500 \mu \mathrm{M}$ ), identified as an antagonist of eugenol on Olfr73 ${ }^{16}$. In contrast to MIEG and isosafrole, di-IEG, as well as a dimer of eugenol (di-Eug, 500 $\mu \mathrm{M}$ ) failed to activate any response in mouse Olfr73-OSNs (Figure 4B, E). Application of the putative antagonists in binary mixture with eugenol also failed to show any inhibition or additivity relative to the control response evoked by eugenol alone (Figure 4C, F). Furthermore, the response to eugenol at its $\mathrm{EC}_{50}$ concentration $(10 \mu \mathrm{M})$ mixed with MIEG $(1 \mathrm{mM})$ was not significantly changed from the control response to eugenol alone (Figure 4D, F), suggesting that pre-incubation with a putative antagonist may impose stronger inhibition. Both dimers, di-Eug and di-IEG $(500 \mu \mathrm{M})$, failed to elicit a response when applied alone for 5 -s (Figure 4B, E). However, pre-incubation for 30-s with di-IEG elicited a small truncated elevation of the GCaMP3 signal, with no attenuation of the response to the binary mix with added eugenol (Figure 4E). Co-application of di-IEG resulted in additive increase of the response to the binary mix with eugenol (Figure 4F), suggesting that the dimers of eugenol and isoeugenol did antagonize the response to eugenol. Overall, we failed to identify any antagonistic or inhibitory interaction of eugenol and its putative antagonists in native mouse OSNs ectopically expressing Olfr73.

\section{In vitro-expressed Olfr73 mediates antagonism between ligands independently of the downstream signaling pathway.}

Since previous research suggested that antagonism in such binary mixtures of ligands may depend on the signaling pathway downstream of the OR in question ${ }^{25}$, we explored the effect of substituting different $\mathrm{G}$ proteins in our in vitro assay. First, we confirmed that mammalian ORs can robustly couple to endogenous stimulatory signaling pathways, mediated not only by Gs/olf but also by Gq11, found in ciliary proteome in mammalian OSNs ${ }^{26}$. Eugenol $(100 \mu \mathrm{M})$ consistently activated calcium release in HEK293 cells co-expressing Olfr73 with the complete heterotrimeric G-protein, Gaq11/ß1/Y13 (Figure 5A). Using this expression system, we then measured the concentration-dependence of the response to eugenol, vanillin and isoeugenol (Figure 5B). Co-expression of another mouse OR, Olfr599 with the heterotrimeric G-protein, Gaq11/ß1/Y13, also conferred robust activation by its cognate ligand, octanoic acid $(100 \mu \mathrm{M})$, as was the case for Gs/olf and Ga15-dependent signaling (Supplemental Figure S3). Thus, mammalian ORs have the capacity of coupling to the heterotrimeric Gq11/b1/Y13 G-protein naturally expressed in olfactory cilia.

We then characterized the interaction between cognate agonists and putative antagonists of rho-tagged Olfr73 co-expressed with either Gaq11/ß1//13, Gs/olf , or the promiscuous G protein, Ga15. We validated the earlier finding that Olfr73 transiently expressed in HEK293 cells conferred the antagonism imposed by 
MIEG on the response to eugenol and another strong agonist, vanillin (Figure 5; Supplemental Figure S4, S5). The response to vanillin $(100 \mu \mathrm{M})$ was only partially inhibited by co-application of MIEG (1 mM), but was completely blocked following 30-s pre-incubation with MIEG (Figure 5D, E; Supplemental Figure S4, S5). Carvone (1 mM), distantly structurally related to eugenol but a non-agonist for Olfr73 ${ }^{27}$ failed to antagonize the response to eugenol even following pre-incubation for 30-s (Figure 5E; Supplemental Figure S4, S5). These findings argue that antagonistic interactions between cognate agonists and putative antagonists can occur independently of which signaling pathway is downstream of ORs expressed in vitro.

\section{Interaction of binary mixtures with the in vitro-expressed Olfr73 is dependent on the stimulus paradigm}

We used the Cre-SEAP assay, similar to the more widely used Cre-Luciferase assay ${ }^{5,9}$ to measure cAMP generated by eugenol applied for a prolonged time to HEK293 cells co-expressing untagged Olfr73 and Gs/olf (Supplemental Figure S6A). MIEG alone also generated cAMP underscoring our finding that in this assay a putative antagonist acts as an agonist (Figure 6A). Surprisingly, we observed no antagonistic interaction between several concentrations of eugenol and MIEG, showing instead an additive effect in net activity (Figure 6A). We used untagged Olfr73 instead of having the receptor rho-tagged on the Nterminus to improve plasma membrane trafficking in assays of in vitro-expressed ORs. In order to address whether the stimulus protocol by itself may have shaped the result, we co-expressed in HEK293 cells the rAAV2/5-targeting plasmid pTR-Olfr73-furin2A-GCaMP3 along with Ga15. In this context eugenol (100 $\mu \mathrm{M}$ ) elicited a robust calcium signal that was significantly diminished after addition of MIEG (1 mM). MIEG alone did not elicit any response (Supplemental Figure S6B,C). From this we assumed that modification of the N-terminus of Olfr73 per se did not affect the OR's ligand binding in vitro nor its coupling to the Gs/olf signaling pathway.

Then, to address the issue of stimulus duration, we measured cAMP-dependent activity in cells coexpressing untagged Olfr73 along with Gs/olf and CNGCmut channel as a cAMP sensor. To ensure that prolonged incubation with ligands did not saturate the cAMP readout, we used isoproterenol $(10 \mu \mathrm{M})$ as a saturating readout. Isoproterenol applied for 5-min activated endogenous adrenergic receptors coupled to Gs/cAMP signaling and generated a robust sustained response exceeding any odorant-evoked response (Figure 6B). Eugenol (100 $\mu \mathrm{M})$ applied for 10-min generated a sustained cAMP-dependent calcium influx (Figure 6B; Supplemental movie S3). A 10-min application of MIEG (1 mM) evoked a more slowly developing cAMP-dependent calcium influx resulting of smaller amplitude (Wilcoxon test, $p=0.018, n=3$ ) (Figure 6B, C). Averaged across several independent measurements, however, steady-state elevation of the cAMP-dependent calcium influx evoked by a binary mixture of eugenol $(100 \mu \mathrm{M})$ and MIEG $(1 \mathrm{mM})$ showed no significant difference from the response evoked by eugenol alone (Wilcoxon test, $p>0.9999, n$ = 4) (Figure 6C; Supplemental movie S4). Thus, two independent methods of detecting Olfr73-activated 
cAMP-dependent activity confirmed that prolonged stimulation of the OR expressed in vitro results in agonism by the same ligand that with brief stimulation is a potent antagonist.

\section{Discussion}

Finding that ligands such as MIEG that were previously characterized as antagonists for Olfr73 expressed in vitro ${ }^{15}$, behave as partial agonists in vivo in native OSNs ectopically expressing the receptor is consistent with previous work. While a subsequent study by the same authors (Oka et al., 2006) failed to find any response induced by MIEG $(300 \mu \mathrm{M})$ in vivo in a limited number of mouse OSNs expressing GFP under the promoter of the endogenous Olfr73, this could reflect the fact that the OSNs were enzymatically isolated from the $\mathrm{OE}$, thus affecting the structural integrity of the cilia and therefore altering the net sensitivity of the OSNs. A more recent study in the same mouse model using electrophysiological patchclamp recording from intact Olfr73-expressing OSNs, found that MIEG behaved as a partial agonist for Olfr73 and failed to antagonize the response to eugenol and another agonist, vanillin, in binary mixtures 28 . Further, imaging of presynaptic odor-evoked activity in the olfactory bulb failed to detect any antagonistic interaction in a binary mixture of eugenol and MIEG ${ }^{29}$. Interestingly, the latter experiment used air-phase odorants that approximated physiological conditions of stimulation. Thus, it appears that in the innate cellular environment, eugenol and MIEG behave as true agonists for ORs sensitive to eugenol, including Olfr73.

Since we can validate the earlier finding that MIEG and structurally similar ligands (isosafrole and dimerized isoeugenol) antagonize the response to eugenol when Olfr73 is expressed in vitro in HEK293 cells ${ }^{15,16}$, we assume that the differences that we found when comparing the results of in vivo expression to the different assay types were not procedural, but instead the result of expression of the receptor in the absence of its native signaling elements. This conclusion is supported by the finding that antagonism between eugenol and MIEG was also observed in a different heterologous in vitro expression system, Xenopus oocytes, in which Olfr73 was co-expressed with $\mathrm{G}_{\text {olf }}$ and a cystic fibrosis transmembrane conductance regulator as a cAMP sensor ${ }^{17}$, and it suggests that ORs can mediate similar responses to ligands in vitro independent of the type of cell used for the expression. This possibility receives further support from our finding that the antagonism measured in vitro was independent of the signaling pathway mediating the agonist-induced response.

The idea that the innate cellular environment critically determines the response properties of an OR aligns with a number of studies of other GPCRs [review: ${ }^{30}$ ], including the finding that pilocarpine, a well-known therapeutic drug and agonist of muscarinic receptors, can act as either an agonist or antagonist on the M3 receptor depending on the cell type, the expression level, and the signaling pathway coupled to the receptor ${ }^{31}$. Moreover, it is now well documented in the GPCR signaling literature that a ligand acting on a single receptor species can impose multiple intrinsic efficacies. In other words, a ligand can be an agonist or antagonist when acting on the same receptor expressed in different cellular environments either in vitro 
or in vivo [review: ${ }^{32}$. An important element that shapes signaling bias is the stochiometric ratio of the different signaling molecules that are able to bind to activated receptor conformation ${ }^{33}$, which one can assume is likely to differ significantly between different cell types and different tissues. Following this logic, the OR-ligand interaction could be governed by a unique complement of endogenous chaperons or guest molecules orchestrating the outcome of the receptor activation in olfactory cilia as proposed earlier for GPCRs in general ${ }^{34}$, especially given that the olfactory cilia constitute a specialized cellular compartment with a highly specialized tubular architecture and distinct plasma membrane lipid composition ${ }^{35}$.

Our study does not address the question of how the ligand-OR interaction plays out to mediate agonism and antagonism. Although ORs can mediate an antagonistic response to ligands independent of the type of in vitro cell suggesting that the $\mathrm{OR}$ is the primary site of the interaction, there is evidence for odorantdependent biased agonism in activation of downstream olfactory signaling pathways. Non-antagonistic ligands for Olfr73 as well as other ORs expressed in vitro act as biased agonists depending on the downstream signaling pathway to which they were coupled ${ }^{25,36}$, and biased agonism in olfactory signaling has been partially documented in vivo ${ }^{37}$. Clearly this question will be important to explore going forward given evidence that non-competitive as well as competitive interactions mediate odorant

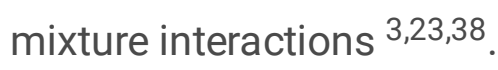

Our finding that the antagonism observed in vitro under short term stimulation was relieved by prolonged stimulation is consistent with prior evidence that Olfr73 manifested a dramatically altered structureactivity relationship in a cAMP-dependent Cre reporter system dependent on the duration of stimulation ${ }^{9}$. It is also consistent with the more general concept that long-term exposure of GPCRs to selective ligands may change occupancy of the receptor and result in different output ${ }^{39}$, which we would suggest can explain the observed time dependency of stimulation. This finding points to the need to not only characterize the pharmacological profile of OR expressed in vivo in native OSNs but also to mimic the natural dynamics of stimulation as closely as possible.

Since natural stimuli are complex odor mixtures, to properly interpret and model representation of the odor space it is critical to understand the actual structure-activity relationships among the ligands comprising these mixtures. Going forward, our findings argue that a combination of ectopic expression of defined ORs in native OSNs in vivo together with high throughput assays of global OSN activity evoked by odorants at near physiological conditions in live animals ${ }^{2,3}$ is likely to provide the most complete understanding of the pharmacological profile and MRR of mammalian ORs. Understanding the mode of operation of ORs in vivo is also increasingly important given that sensory GPCRs have been directly implicated in regulating health conditions in a number of non-olfactory tissues ${ }^{40}$.

\section{Methods}

\section{Animals}


Adult 4 to 8-week old C57/BI6 mice and Sprague-Dawley rats of both sexes were used in the study. All procedures were performed in accordance with the ARRIVE guidelines and the University of Florida IACUCapproved protocol and in accordance with the National Institutes of Health guidelines.

\section{rAAV2/5 design and production}

To engineer the rAAV5 based bicistronic expression system we used the previously described plasmid pTR UF50-BC encoding a fluorescent protein mApple and Firefly Luciferase ${ }^{41}$. Two separate ORexpressing cassettes were designed: Olfr73-furin-F2A-GCaMP3, and Olfr599-furin-F2A-GCAMP, each subcloned between two AAV2 ITRs. Full-length Olfr599 (a gift from Dr. Matsunami, Duke University) and Olfr73 (a gift from Dr. Touhara, Tokyo University) were cloned in-frame with a furin protease target cleavage peptide fused to a ribosome-skipping sequence 2A, immediately followed by GCaMP3 reporter. The GCaMP3-encoding sequence was amplified by PCR from a lipid-anchored version Lck-GCaMP3 (Addgene \#26974, a gift from Dr. Shigetomi). The cassette contained no additional sequence tags enhancing surface targeting of the OR, ensuring that both co-expressed open-reading frame (ORF) sequences were synthesized as precise phenocopies of their wild type counterparts. Expression of the bicistronic transcript was driven by a strong ubiquitous chicken $\beta$-actin promoter containing cytomegalovirus transcription enhancer element. Standard subcloning technique and Gibson assembly (New England Biolabs) were used to assemble inserts. All sequences were independently verified by a commercial service (IDT Inc.). For packaging rAAV5, a plasmid pXYZ5, containing all necessary helper viral genes along with the AAV vector plasmid were transfected into HEK293 cells. Following $72 \mathrm{~h}$ posttransfection, cells were harvested and rAAV5 was purified using an iodixanol gradient method described earlier ${ }^{19}$.

\section{In Vivo Imaging of Bioluminescence}

Mice were anesthetized with a Ketamine/Xylazine mixture and 10-15 $\mu \mathrm{L}$ of rAAV-CBA-mApple-furin2ALuciferase (titer ca. $10^{11} \mathrm{vg} / \mathrm{ml}$ ), was delivered intranasally as a single infusion per nostril. Animals were used for imaging at least 7 days post-inoculation as described previously ${ }^{42}$. As a control for in vivo Luciferase imaging, a small volume of the vector was injected in the tibial muscle of hind leg. For in vivo imaging, animals were anesthetized with isoflurane, intraperitoneally injected with $15 \mathrm{mg} / \mathrm{mL}$ of XenoLight potassium salt of D-luciferin (PerkinElmer) dissolved in sterile divalent-free sterile PBS, and imaged between 5 and 20 min after the injection using a Xenogen IVIS imager (Perkin Elmer). The images were analyzed and saved as TIFF files using Living Image software (PerkinElmer). Following in vivo imaging the same animal was cardiac perfused with the ice-cold 4\% PFA in phosphate-buffered saline. The nasal tissue was decalcified, protected in $30 \%$ sucrose and frozen in OCT medium. Cryostat coronal sections were examined for the presence of mApple fluorescence in the olfactory epithelium.

\section{En face confocal imaging of the olfactory epithelium}


Animals were anesthetized with $\mathrm{CO}_{2}$, rapidly decapitated, and the entire turbinates and septum were dissected and kept on ice in a petri dish filled with freshly oxygenated with carbogen modified artificial cerebrospinal fluid (ACSF) that contained (in mM): $120 \mathrm{NaCl}, 25 \mathrm{NaHCO}_{3}, 3 \mathrm{KCl}, 1.25 \mathrm{Na}_{2} \mathrm{HPO}_{4}, 1 \mathrm{MgSO}_{4}$, $1.8 \mathrm{CaCl}_{2}, 15$ glucose, $305 \mathrm{mOsm}$ (adjusted with sucrose), $\mathrm{pH} 7.4$. For imaging, a small piece of the olfactory epithelium was mounted in a perfusion chamber (RC-23, Warner Instruments) with the apical surface facing down and analyzed in a Leica SP5 confocal microscope equipped with a 63x waterimmersion objective, using a preset configuration for acquisition of mApple fluorescence.

\section{Single cell GCaMP3 calcium imaging}

Animals 4-6 weeks of age were used for experiments $7-21$ days post-inoculation with rAAV2/5 encoding OR-furin2A-GCaMP3. Calcium imaging was performed as detailed previously ${ }^{22}$. Tissue was prepared and mounted with the apical surface facing up. The chamber was transferred to the stage of an upright microscope (Zeiss Axioskop-2F) equipped with a 40x/0.75NA long distance water-immersion objective lens. Experimental solutions were applied directly to the field of view through a $100 \mu \mathrm{m}$ diameter needle made of fused silica and connected to a 9-channel Teflon manifold. Solution application was controlled by electronic valves (VC-6, Warner Instruments). The calcium response is presented as an increase of GCaMP3 fluorescence originating from the knob and underlying dendrite. The tissue was illuminated using a standard eGFP filter cube BP490 nm/ $535 \mathrm{~nm}$ (Omega Optical, USA) and the emitted light was collected at 530anm (BP 530/20 nm, Omega Optical, USA) by a 12-bit cooled CCD camera (ORCA R2, Hamamatsu, Japan). Both the illumination system (Lambda DG-4, Sutter Instruments, USA) and image acquisition were controlled by Imaging Workbench 6 software (INDEC BioSystems). Before processing, fluorescence intensity was corrected for the background. Each knob was assigned a region of interest (ROI) and changes in fluorescence intensity within each ROI were analyzed and expressed as the peak fractional change in fluorescent light intensity (F-Fo)/Fo where Fo is the baseline fluorescence before application of experimental solutions.

\section{Transient expression of mouse ORs in HEK293 cells}

Heterologous expression of mouse ORs was based on the previously published method ${ }^{36}$. HEK293 (ATCC CRL-1537) or HEK293T (ATCC CRL-3216) cells were grown in a high glucose Dulbecco's minimum essential medium (DMEM) supplemented with $10 \%$ FBS, penicillin and streptomycin, and $2 \mathrm{mM} \mathrm{L-}$ glutamine and maintained at $37^{\circ} \mathrm{C}\left(5 \% \mathrm{CO}_{2}\right)$. A rhodopsin-tagged mouse olfactory receptor Olfr 73 and Olfr599 (provided by Dr. Matsunami, Duke University) was co-expressed with RTP1s, a short version of the Receptor Transporting Protein 1 ensuring proper surface trafficking of the receptor (provided by Dr. Touhara, University of Tokyo). To reconstitute different G-protein coupled signaling pathways, one of the three G-proteins was co-expressed along with the OR and RTP1s, promiscuous mouse $\mathrm{G}_{\mathrm{a}} 15$ (Thermo Scientific Open Biosystems), heterotrimeric $G_{a} q 11 / ß 1 / \gamma 13$, both coupled to endogenous PLC/calcium release, and human $\mathrm{G}_{\mathrm{a}}$ olf (Missouri S\&T cDNA Resource Center). To assay activity-dependent accumulation of cAMP generated by activation of the Gs/Golf pathway, the cells were additionally cotransfected with a mutated A2 subunit of the rat olfactory cyclic nucleotide gated channel (CNGCmut). 
Two mutations C460W and E583M ensured high sensitivity and selectivity of the channel to cAMP (a gift from Dr. Rich, University of South Alabama). Transfections were performed using Calfectin (SignaGen). Cells at $70-80 \%$ confluence were co-transfected with equimolar ratios of the OR plasmid, RTP1s, the plasmid encoding each subunit of the G-protein in question, and the CNGCmut-encoding plasmid. To assay potential effects of the $\mathrm{N}$-terminal modification on OR function, the cells were separately transfected with untagged version of the same OR encoded by the rAAV5 plasmid pTR-CBA-OR-furin-2AGCaMP3. After transfection, the cells were grown for at least $48 \mathrm{~h}$ to allow the functional reconstitution of the cAMP-dependent pathway and the CNGCmut reporter assay.

\section{Calcium imaging of odorant induced response in HEK293 cells}

Calcium imaging was performed as described previously ${ }^{36}$. HEK293 cells were incubated $\left(30 \mathrm{~min} / 37^{\circ} \mathrm{C}\right)$ in a DMEM containing 6-8 $\mu$ M Fluo-4/AM (Invitrogen) or Fluo-2/AM (TefLabs) containing $0.04 \%$ Pluronic F127. Activity of cells expressing untagged ORs from the bicistronic pTR plasmid was reported by expressed GCaMP3. Odorant solutions were applied sequentially to the cells for $5 \mathrm{~s}$ with a 3 min interval between each application to allow recovery from desensitization resulting from previous application of odorants. In the case of prolonged 10-min stimulation, the cells were allowed to recover for at least 10 min before adding a binary mix or isoproterenol alone, activating endogenous production of cAMP. Cells growing either on a 35-mm plastic dish or on a round poly-L-lysine coated cover glass were transferred to the stage of an inverted microscope (Axiovert 200, Zeiss) equipped with a 10x/0.5NA Fluar objective. Odorants were applied to the cells using a computer-controlled fast perfusion system (RSC-200, BioLogic, France) ensuring precise delivery of the stimulus with a minimal time lag. The perfusion system was controlled by Clampex 9.2 or 10.2 software (Molecular Devices). Additional measurements were performed using an inverted microscope (Olympus IX-71) equipped with a 20x/0.45NA U-Plan objective lens. Both microscopes were equipped with 12-bit cooled CCD cameras (ORCA R2, Hamamatsu, Japan). A standard FITC filter set (excitation at $510 \mathrm{~nm}$, emission at $530 \mathrm{~nm}$, dichroic mirror $516 \mathrm{~nm}$ ) was used for single-wavelength measurements. The illumination system (Lambda DG-4 or Lambda L10-BS with a Smart Shutter controller, Sutter Instruments) and image acquisition were controlled by Imaging Workbench 6 software (INDEC BioSystems) under master control of Clampex software (Molecular Devices) to ensure syncing of image acquisition and odorant application. Image post-processing was performed as described above.

\section{Using a Cre-SEAP assay to measure cAMP production activated by odorants}

cAMP production was measured as previously described ${ }^{43}$. HEK293T cells were co-transfected with a guanine nucleotide exchange factor Ric-8b (50 ng; a gift from Dr. Malnic, Universidade de São Paulo, Brazil), Gaolf (50 ng), RTP1s (100 ng) and untagged Olfr73 $(1.5 \mu \mathrm{g})$. Cells were also transfected with $1.5 \mu \mathrm{g}$ of a PCRE-SEAP, where the expression of the secreted alkaline phosphatase (SEAP) is under regulation of the cAMP responsive element. A plasmid pTAL-SEAP missing the Cre element was used as 
a negative control. Cells were also transfected with $50 \mathrm{ng}$ of a pcDNA5/TO/LacZ (Invitrogen) for DNA concentration normalization. At $24 \mathrm{~h}$ post-transfection, the cells were re-seeded for SEAP analysis and odorants were added at the indicated dilutions at $48 \mathrm{~h}$ post-transfection. Cells and supernatants were collected $20 \mathrm{~h}$ later and centrifuged for $5 \mathrm{~min}$ at $5,000 \mathrm{~g}$. The supernatants were incubated for $30 \mathrm{~min}$ at $65^{\circ} \mathrm{C}$ and then frozen until analysis. The cell pellets were washed with PBS and then lysed with reporter

lysis buffer for $\beta$-galactosidase measurement following the manufacturer's instructions (Promega). SEAP activity was measured by mixing $100 \mu \mathrm{l}$ of supernatant with an equal amount of BluePhos substrate (KPL). Samples were monitored for color development at $630 \mathrm{~nm}$ in a microwell plate reader. SEAP activity was calculated by subtracting the responses of cells transfected in parallel with PTAL-SEAP and normalizing to $\beta$-gal activity. Average SEAP activity was determined after subtracting the response of cells not expressing an OR and is reported in arbitrary units \pm standard deviation.

\section{Reagents, odorants and solution application}

IBMX, forskolin, and SQ23385 (from Tocris or Cayman) were dissolved in DMSO and stocks kept at $-20^{\circ} \mathrm{C}$. Single odorants of the highest purity were purchased from Sigma-Aldrich, Acros Chemicals, Alpha Aesar and dissolved in anhydrous DMSO as a $0.5 \mathrm{M}$ stock. For application odorants were delivered as aqueous solutions at the concentration indicated, prepared in freshly oxygenated ACSF. ACSF supplemented with $0.1 \%$ DMSO, the odorant carrier, served as the control solution. Odorant stocks were kept at $-20^{\circ} \mathrm{C}$ and the final aqueous solutions were prepared on the day of the experiment in a magnesium-free Ringer's solution containing (in $\mathrm{mM}$ ): $140 \mathrm{NaCl}, 5 \mathrm{KCl}, 1.8 \mathrm{CaCl}_{2}, 0 \mathrm{MgCl}_{2}, 10 \mathrm{HEPES}$, 1.25 sodium pyruvate, 10 glucose, $\mathrm{pH} 7.6$, and diluted immediately before experiments to the indicated concentration.

\section{Statistical analysis}

Analysis and graphical presentation of the data were performed with Imaging Workbench 6 (INDEC), Microsoft Excel, Clampfit 9.2 (Molecular Devices), NIH ImageJ 1.52 (http://imagej.nih.gov/ij) and assembled in CorelDraw v.18 (Corel). Statistical analysis was performed in Microsoft Excel and Graph Pad Prism 8.

\section{Declarations}

\section{Acknowledgements}

Special thanks to Drs. Patricia Duchamp-Viret and Johannes Reisert for critical reading an earlier draft of the manuscript. Chemical dimers of isoeugenol and eugenol were generously provided by Dr.Renzo Bortolomeazzi (University of Udine, Italy).

\section{Funding}


This work was supported by the National Institutes of Health through awards DC011859 to BWA and DC012819 to SZ.

\section{Author contributions}

$\mathrm{KU}$ (cloning, making AAV, collecting and analyzing data), EAC (cloning, collecting and analyzing data), SZ (writing the manuscript), BWA (writing the manuscript). All authors reviewed and approved the final version of the manuscript.

\section{Competing interests}

Authors declare no competing interests

\section{References}

1. Zhang, X. \& Firestein, S. The olfactory receptor gene superfamily of the mouse. Nat Neurosci $5,124-$ 133 (2002).

2. Zak, J. D., Reddy, G., Vergassola, M. \& Murthy, V. N. Antagonistic odor interactions in olfactory sensory neurons are widespread in freely breathing mice. Nat. Commun. 11, 3350 (2020).

3. Inagaki, S., Iwata, R., Iwamoto, M. \& Imai, T. Widespread Inhibition, Antagonism, and Synergy in Mouse Olfactory Sensory Neurons In Vivo. Cell Rep. 31, 107814 (2020).

4. Xu, L. et al. Widespread receptor-driven modulation in peripheral olfactory coding. Science (80- .). 368, eaaz5390 (2020).

5. Zhuang, H. \& Matsunami, H. Evaluating cell-surface expression and measuring activation of mammalian odorant receptors in heterologous cells. Nat Protoc 3, 1402-1413 (2008).

6. McIntyre, J. C., Hege, M. M. \& Berbari, N. F. Trafficking of ciliary G protein-coupled receptors. Methods Cell Biol. 132, 35-54 (2016).

7. Krautwurst, D., Yau, K. W. \& Reed, R. R. Identification of ligands for olfactory receptors by functional expression of a receptor library. Cell 95, 917-926 (1998).

8. Saito, H., Chi, Q., Zhuang, H., Matsunami, H. \& Mainland, J. D. Odor coding by a Mammalian receptor repertoire. Sci Signal 2, ra9 (2009).

9. Baud, O. et al. The mouse eugenol odorant receptor: structural and functional plasticity of a broadly tuned odorant binding pocket. Biochemistry 50, 843-853 (2011).

10. Wachowiak, M. All in a Sniff: Olfaction as a Model for Active Sensing. Neuron 71, 962-973 (2011).

11. Bozza, T., Feinstein, P., Zheng, C. \& Mombaerts, P. Odorant receptor expression defines functional units in the mouse olfactory system. J. Neurosci. 22, 3033-3043 (2002).

12. Dewan, A. et al. Single olfactory receptors set odor detection thresholds. Nat. Commun. 9, 1-12 (2018). 
13. Zhao, H. et al. Functional expression of a mammalian odorant receptor. Science (80- ). 279, 237242 (1998).

14. Stein, B., Alonso, M. T., Zufall, F., Leinders-Zufall, T. \& Chamero, P. Functional overexpression of vomeronasal receptors using a herpes simplex virus type 1 (HSV-1)-derived amplicon. PLoS One 11, 1-13 (2016).

15. Oka, Y., Omura, M., Kataoka, H. \& Touhara, K. Olfactory receptor antagonism between odorants. EMBO J 23, 120-126 (2004).

16. Oka, Y., Nakamura, A., Watanabe, H. \& Touhara, K. An odorant derivative as an antagonist for an olfactory receptor. Chem. Senses 29, 815-22 (2004).

17. Abaffy, T., Matsunami, H. \& Luetje, C. W. Functional analysis of a mammalian odorant receptor subfamily. J Neurochem $97,1506-1518$ (2006).

18. Castle, M. J., Turunen, H. T., Vandenberghe, L. H. \& Wolfe, J. H. Controlling AAV tropism in the nervous system with natural and engineered capsids. in Methods in Molecular Biology 1382, 133-149 (Humana Press Inc., 2016).

19. Zolotukhin, S. et al. Production and purification of serotype 1, 2, and 5 recombinant adenoassociated viral vectors. Methods 28, 158-167 (2002).

20. Saito, H., Kubota, M., Roberts, R. W., Chi, Q. \& Matsunami, H. RTP family members induce functional expression of mammalian odorant receptors. Cell 119, 679-691 (2004).

21. Reisert, J. Origin of basal activity in mammalian olfactory receptor neurons. J Gen Physio/ 136, 529540 (2010).

22. Ukhanov, K. Y., Bobkov, Y. V., Martens, J. R. \& Ache, B. W. Initial Characterization of a Subpopulation of Inherent Oscillatory Mammalian Olfactory Receptor Neurons. Chem. Senses 44, 583-592 (2019).

23. Rospars, J.-P., Lansky, P., Chaput, M. \& Duchamp-Viret, P. Competitive and Noncompetitive Odorant Interactions in the Early Neural Coding of Odorant Mixtures. J. Neurosci. 28, 2659-2666 (2008).

24. Neubig, R. R., Spedding, M., Kenakin, T. \& Christopoulos, A. International Union of Pharmacology Committee on Receptor Nomenclature and Drug Classification. XXXVIII. Update on terms and symbols in quantitative pharmacology. Pharmacol Rev 55, 597-606 (2003).

25. Shirokova, E. et al. Identification of specific ligands for orphan olfactory receptors. G proteindependent agonism and antagonism of odorants. J Biol Chem 280, 11807-11815 (2005).

26. Kuhlmann, K. et al. The membrane proteome of sensory cilia to the depth of olfactory receptors. Mol. Cell. Proteomics 13, 1828-1843 (2014).

27. Repicky, S. E. \& Luetje, C. W. Molecular receptive range variation among mouse odorant receptors for aliphatic carboxylic acids. J Neurochem 109, 193-202 (2009).

28. Scholz, P. et al. Identification of a novel Gnao-mediated alternate olfactory signaling pathway in murine OSNs. Front. Cell. Neurosci. 10, (2016).

29. Cruz, G. \& Lowe, G. Neural coding of binary mixtures in a structurally related odorant pair. Sci. Rep. 3, $1-11$ (2013). 
30. Michel, M. C. \& Charlton, S. J. Biased agonism in drug discovery-is it too soon to choose a path? in Molecular Pharmacology 93, 259-265 (American Society for Pharmacology and Experimental Therapy, 2018).

31. Pronin, A. N., Wang, Q. \& Slepak, V. Z. Teaching an old drug new tricks: Agonism, antagonism, and biased signaling of pilocarpine through M3 muscarinic acetylcholine receptor. Mol. Pharmacol. 92, 601-612 (2017).

32. Berg, K. A. \& Clarke, W. P. Making Sense of Pharmacology: Inverse Agonism and Functional Selectivity. Int. J. Neuropsychopharmacol. 21, 962-977 (2018).

33. Onfroy, L. et al. G protein stoichiometry dictates biased agonism through distinct receptor-G protein partitioning. Sci. Rep. 7, (2017).

34. Kenakin, T. \& Christopoulos, A. Measurements of ligand bias and functional affinity. Nat. Rev. Drug Discov. 12, 483 (2013).

35. Verhey, K. J. \& Yang, W. Permeability barriers for generating a unique ciliary protein and lipid composition. Current Opinion in Cell Biology 41, 109-116 (2016).

36. Ukhanov, K., Bobkov, Y., Corey, E. A. \& Ache, B. W. Ligand-selective activation of heterologouslyexpressed mammalian olfactory receptor. Cell Calcium 56, 245-256 (2014).

37. Ukhanov, K., Brunert, D., Corey, E. A. \& Ache, B. W. Phosphoinositide 3-kinase-dependent antagonism in mammalian olfactory receptor neurons. J. Neurosci. 31, 273-80 (2011).

38. Ache, B. W. Position Review: Functional Selectivity in Mammalian Olfactory Receptors. Chem. Senses 45, 503-508 (2020).

39. Vauquelin, G. \& Charlton, S. J. Long-lasting target binding and rebinding as mechanisms to prolong in vivo drug action. British Journal of Pharmacology 161, 488-508 (2010).

40. Lee, S. J., Depoortere, I. \& Hatt, H. Therapeutic potential of ectopic olfactory and taste receptors. Nature Reviews Drug Discovery 18, 116-138 (2019).

41. Marsic, D., Méndez-Gómez, H. R. \& Zolotukhin, S. High-accuracy biodistribution analysis of adenoassociated virus variants by double barcode sequencing. Mol. Ther. - Methods Clin. Dev. 2, 15041 (2015).

42. Williams, C. L. et al. Gene Therapeutic Reversal of Peripheral Olfactory Impairment in Bardet-Biedl Syndrome. Mol. Ther. 25, (2017).

43. Durocher, Y. et al. A reporter gene assay for high-throughput screening of G-protein-coupled receptors stably or transiently expressed in HEK293 EBNA cells grown in suspension culture. Anal. Biochem. 284, 316-26 (2000).

\section{Figures}



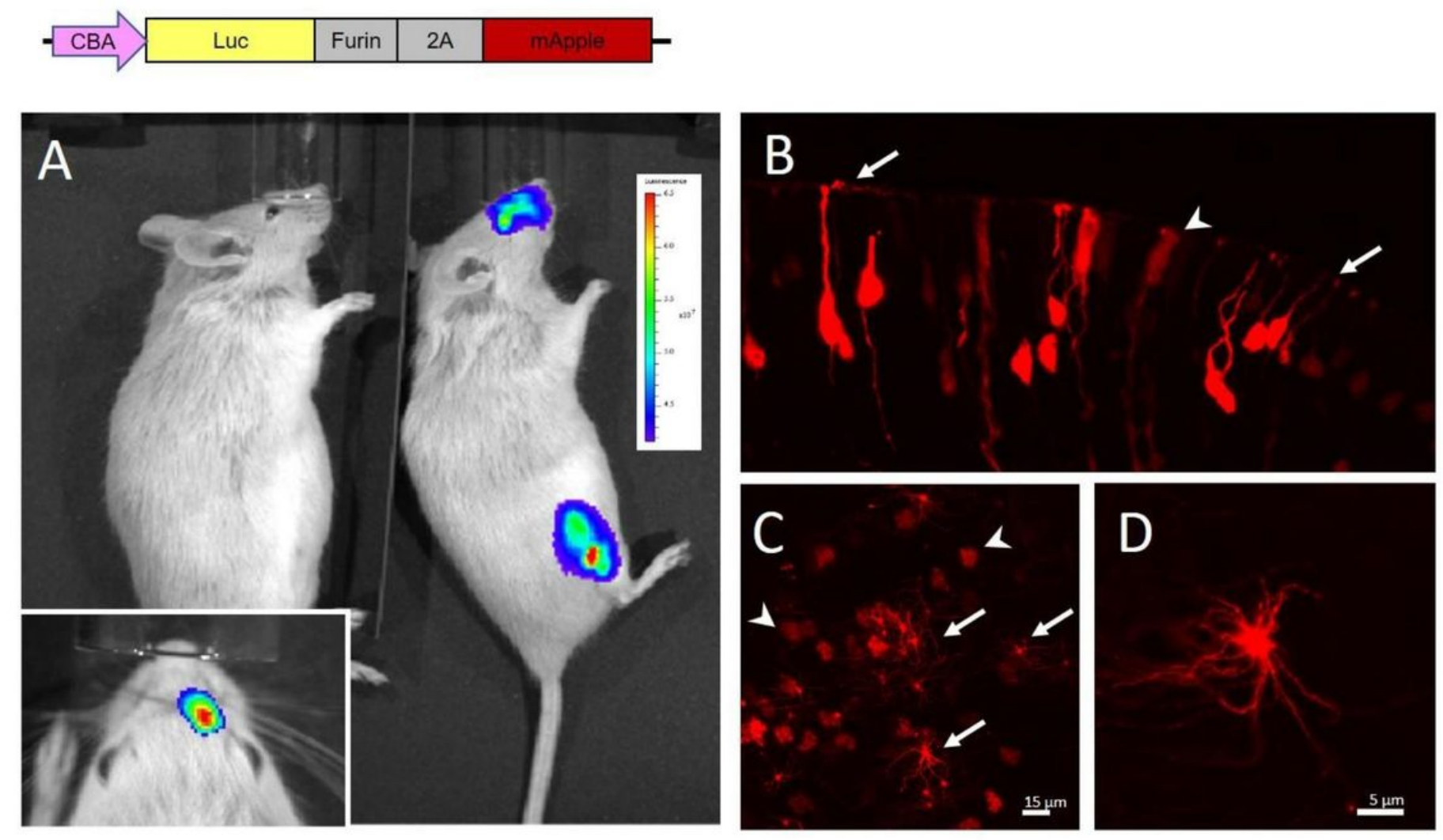

\section{Figure 1}

rAAV2/5 assisted gene delivery of Luciferase-furin2A-mApple induces persistent expression of the reporter proteins. (A) A single $20 \mu \mathrm{L}$ injection of the rAAV2/5 vector induced ectopic expression of luciferase in the nasal cavity and tibial muscle. (Insert) Unilateral intranasal infusion of the vector confirmed restricted expression of the reporter. Control injection of sterile saline was used as a negative control (left animal). Pseudo-color scale shows intensity of luciferase bioluminescence from 3 to $6.5 * 107$ photons per second. (B) Coronal section of postfixed OE from the same animal used for in vivo imaging in (A). rAAV2/5 induced expression of mApple in OSNs (arrows) and in sustentacular cells (arrowheads). (C) En face images of the freshly dissected OE showing groups of rAAV2/5 induced OSNs (arrows) and non-sensory sustentacular cells (arrowheads). (D) Magnified image of the dendritic knob decorated with numerous cilia. 

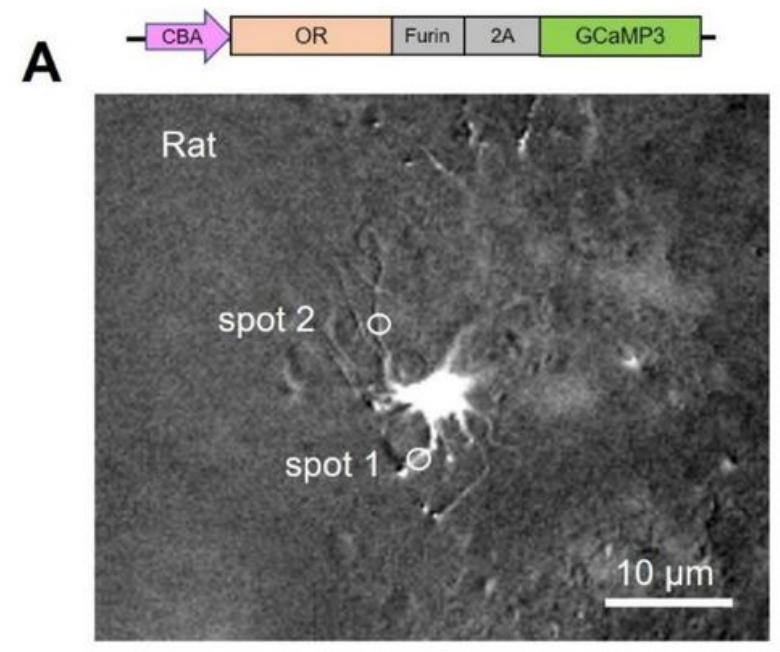

C

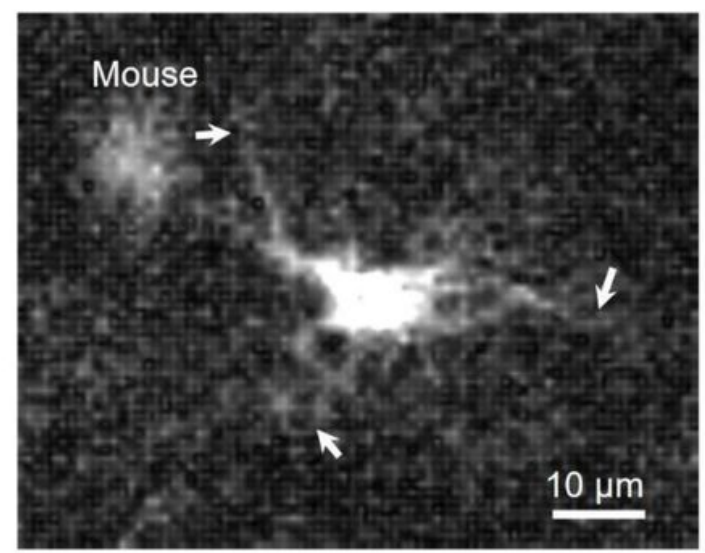

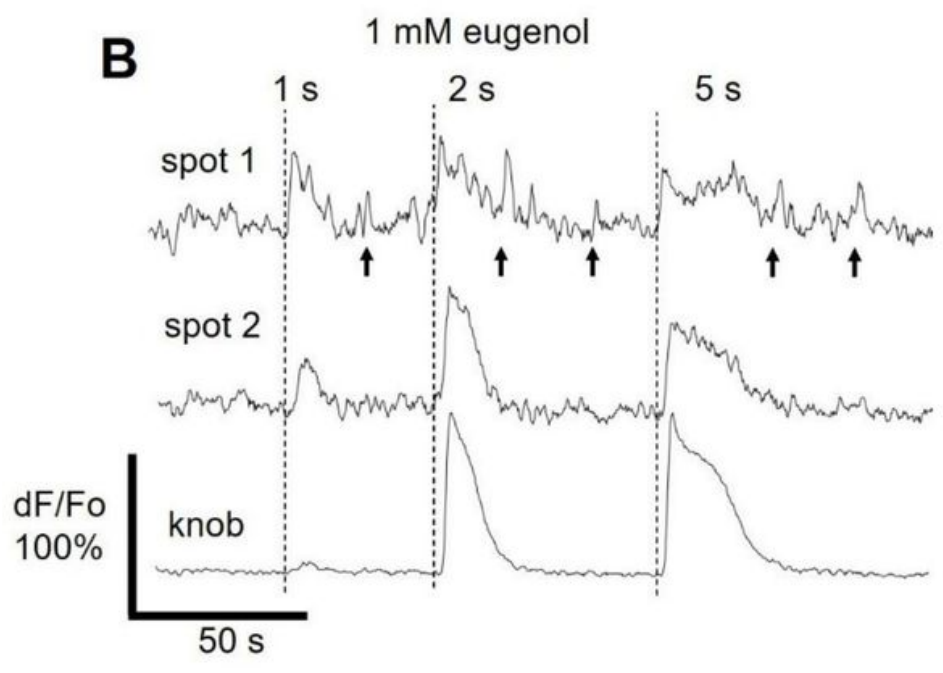

D

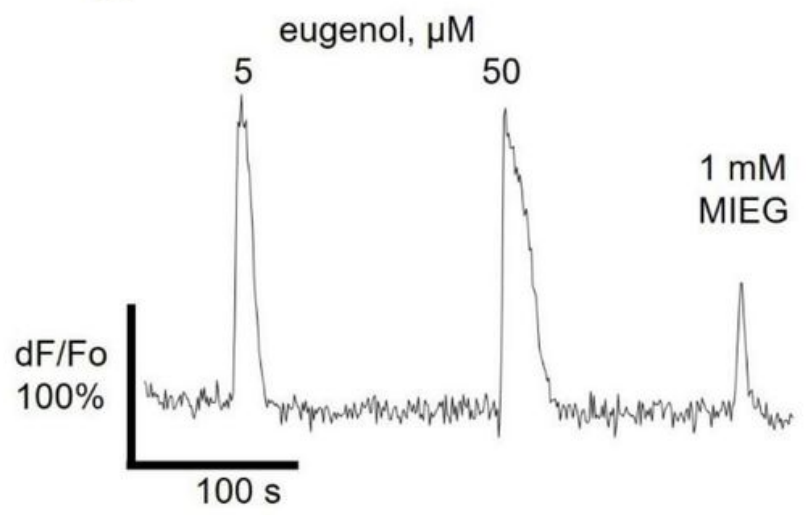

\section{Figure 2}

Robust eugenol-evoked activation of mammalian OSNs ectopically expressing Olfr73. Neuronal activity was measured with GCaMP3 expressed from the same rAAV2/5 vector along with the OR. $(A, B)$ A single knob of the rat Olfr73-OSN which was stimulated with a solution containing $1 \mathrm{mM}$ eugenol with pulses of increasing duration. A short 1-s long pulse activated nearly threshold response in the knob (bottom trace). However, in two representative cilia the same stimulus elicited much stronger relative increase of GCaMP3 fluorescence (spot 1 and 2). Peristimulus activity persisted as reflected by a transient intraciliary $\mathrm{Ca} 2+$ fluctuations (arrows, upper trace). (C,D) Mouse Olfr73-OSN was strongly activated by a low concentration of eugenol ( $5 \mu \mathrm{M}$, 5-s pulse). Application of methylisoeugenol (MIEG, $1 \mathrm{mM}, 5$-s pulse) also elicited a response. Cilia attached to the knob can be clearly resolved (arrows). 

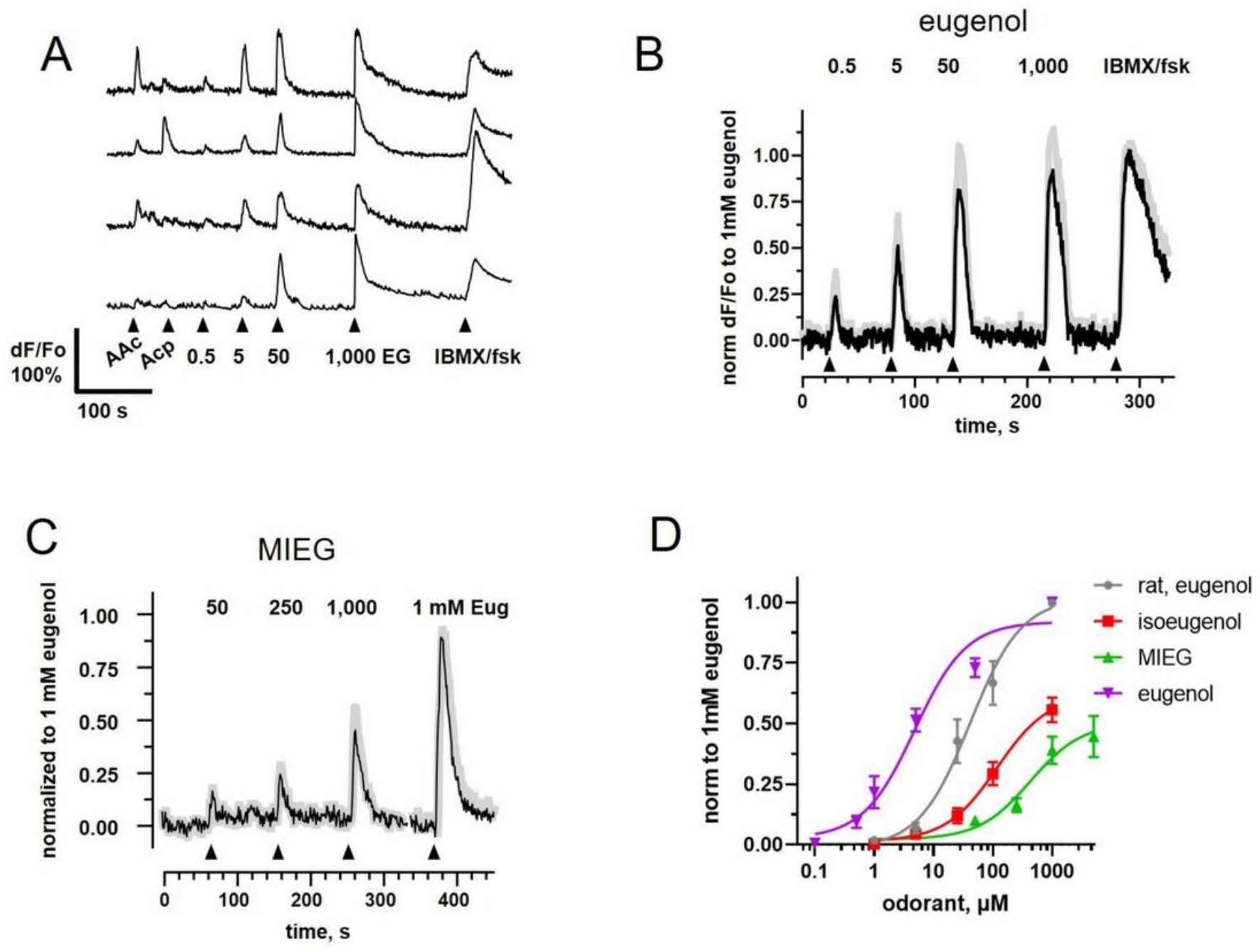

Figure 3

Characterization of the response to eugenol measured in Olfr73-GCaMP3-OSNs in mouse OE. (A) Typical profile of the response elicited by application of two distinct from eugenol odorants, amyl acetate (AAc) and acetophenone (Acp). Each of the selected OSNs reacted differently to AAc and Acp (each at $100 \mu \mathrm{M}$ ). However, increasing concentration of eugenol evoked a dose-dependent response in all 11 cells in this group. A solution containing IBMX/forskolin (IBMX/fsk, 100/10 $\mu \mathrm{M}$ ) was used as a positive control activating functional canonical OSNs. (B, C) The response measured in cells sequentially stimulated with increasing concentration of eugenol, followed by MIEG (concentration of ligand in $\mu \mathrm{M}$ is indicated above each trace). After applying MIEG, a pulse of eugenol (Eug, $1 \mathrm{mM}$ ) was given as a positive control. (D) Summary of the dose-response profiles of the mouse OSNs. Data points were fitted to the Hill equation yielding the following EC50 values $(41 \pm 12 \mu \mathrm{M}$ eugenol, rat, $\mathrm{n}=13 ; 4.8 \pm 1.1 \mu \mathrm{M}$ eugenol, $\mathrm{n}=24 ; 117 \pm 45$ $\mu \mathrm{M}$, isoeugenol, $\mathrm{n}=24 ; 430 \pm 230 \mu \mathrm{M}, \mathrm{MIEG}, \mathrm{n}=15)$. The data were collected from 6 mice and 2 rats. 

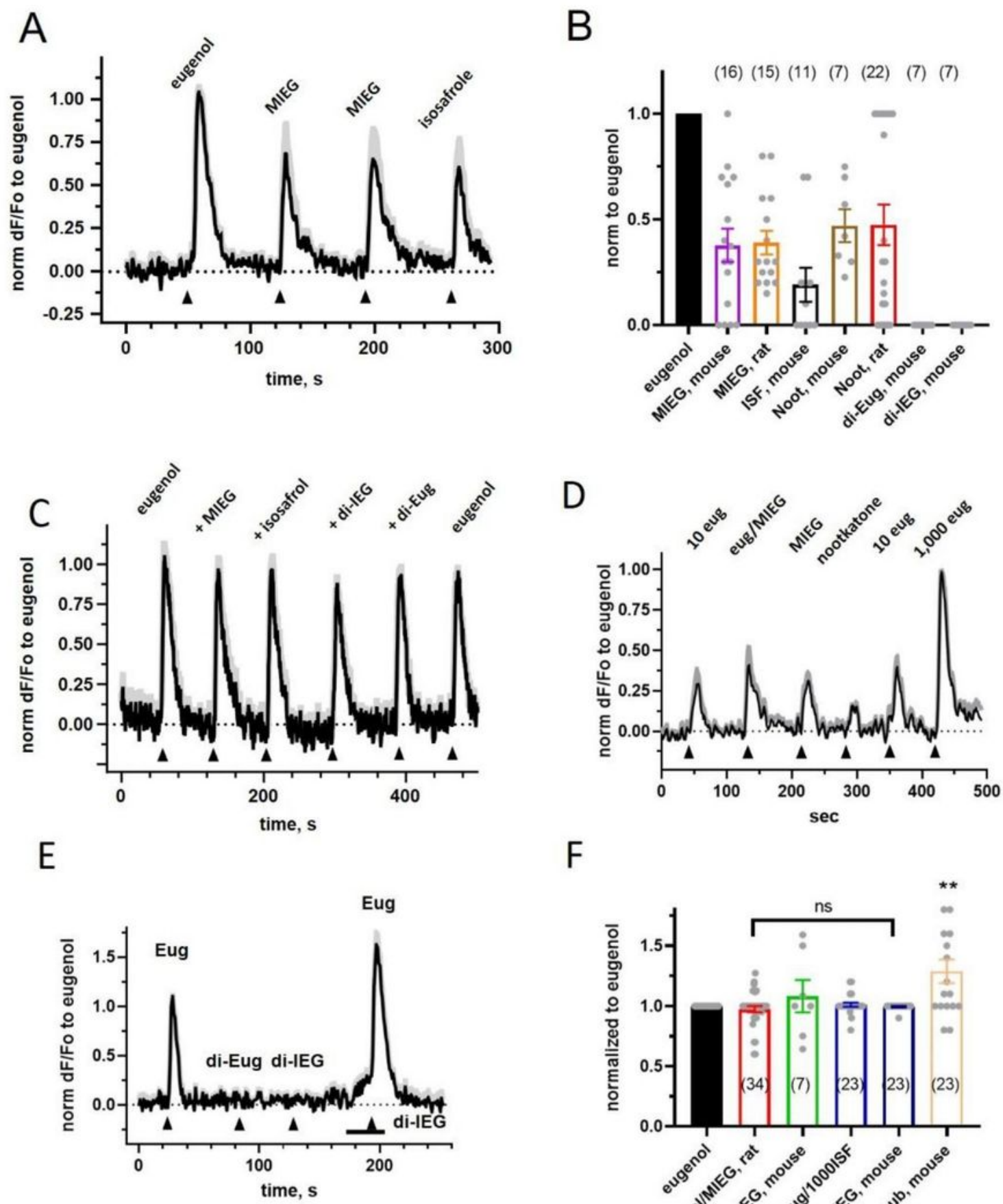

$\mathrm{F}$

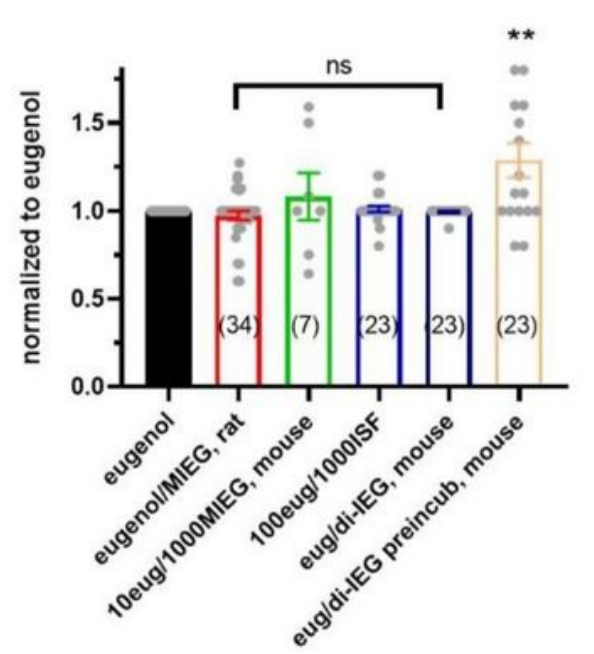

\section{Figure 4}

Rodent Olfr73-GCaMP3-OSNs were probed with a single concentration of eugenol and a known antagonist. (A) Mouse OSNs activated by eugenol (100 $\mu \mathrm{M})$, MIEG (1 mM) and isosafrole (1 mM). (B) Response elicited by each ligand was normalized to that of eugenol $(100 \mu \mathrm{M})$. MIEG and nootkatone similarly activated mouse and rat OSNs. Dimers of eugenol (di-EG) and isoeugenol (di-IEG) applied at 500 $\mu \mathrm{M}$ did not elicit any response. Number of cells in each set is show above each bar. (C) Binary mix of 
eugenol $(100 \mu \mathrm{M})$ and a known antagonist (same concentration as in B) was applied to mouse OSNs. After the test, eugenol $(100 \mu \mathrm{M})$ was applied again to account for any run-down. (D) High concentration of MIEG (1 mM) mixed with low $10 \mu \mathrm{M}$ eugenol evoked the same amplitude of the response as eugenol alone. Total of 5 cells were measured. (E) Dimer of isoeugenol was not able to antagonize the response elicited by eugenol $(100 \mu \mathrm{M})$ following preincubation for 30 -s (bar below the trace). Pre-incubation with di-IEG evoked a small increase of GCaMP3 signal. (F) Response evoked by different binary mixes were normalized to that evoked by eugenol alone. Wilcoxon paired test, ${ }^{\star} \mathrm{p}=0.017$. Number of cells used in each set is shown inside bars. $(A, C, E)$ Averaged traces are shown with SEM overlaid as a gray shadow.
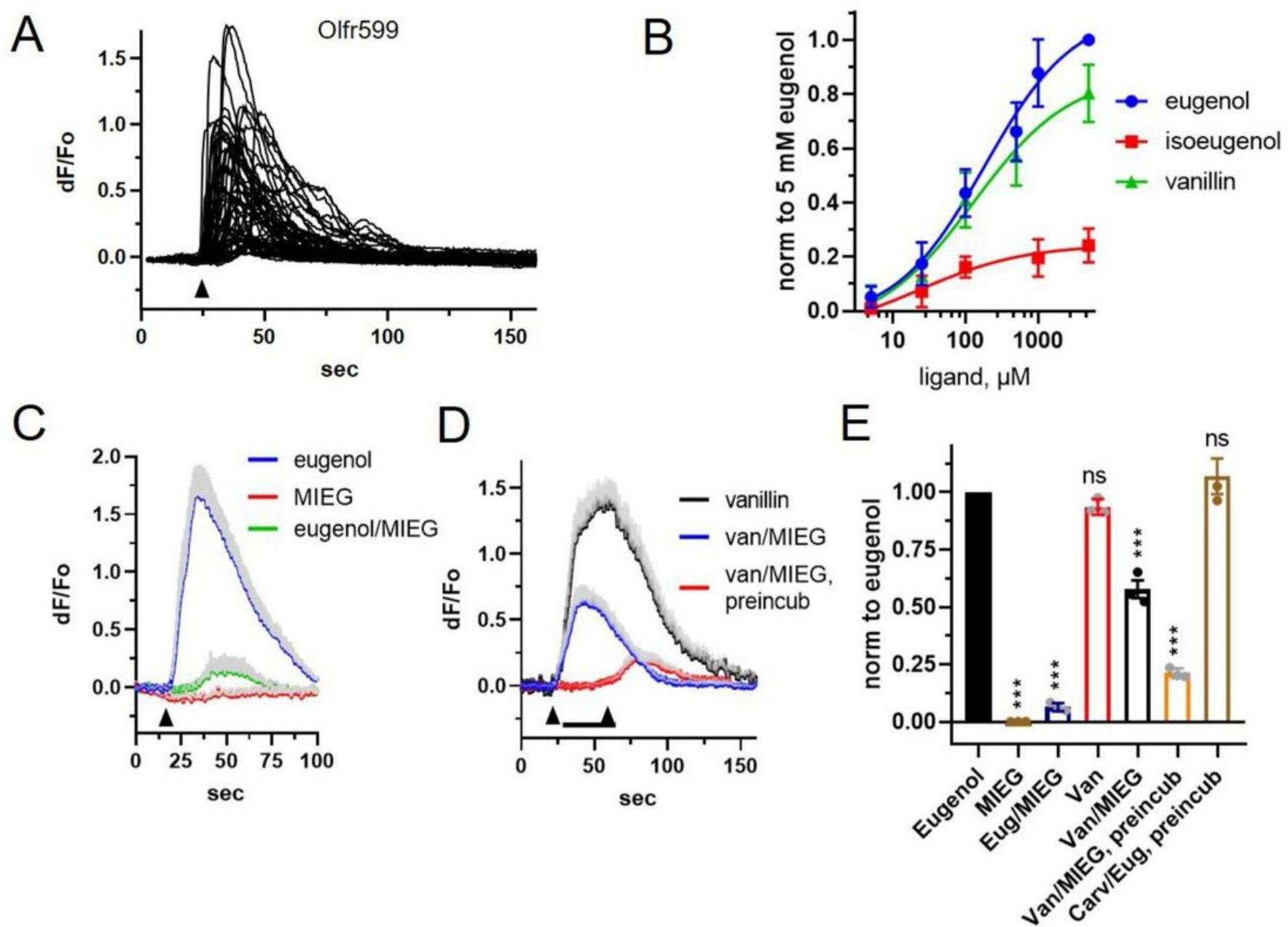

Figure 5

Co-expression of Olfr73 with a native heteromeric G-protein $\mathrm{Gq} 11 / \mathrm{b} 1 / \mathrm{g} 13$ does not change the antagonism in the binary mix of agonists and putative antagonists. (A) Co-expression of Olfr73 with a complete heterotrimeric G-protein Gaq11 couples the OR to the endogenous PLC-dependent calcium release following a 5-s application of eugenol $(100 \mu \mathrm{M})$ indicated by arrow below the traces. The eugenolevoked response was measured with Fluo-3 individually in 25 cells. (B) Concentration-dependence of the response elicited by three Olfr73 agonists. The response represents changes of GCaMP3 fluorescence 
normalized to that evoked by $5 \mathrm{mM}$ eugenol. Data points were fitted to the sigmoidal curve yielding the following EC50 values (184 $\pm 62 \mu \mathrm{M}$, eugenol; $23 \pm 8 \mu \mathrm{M}$, isoeugenol; $130 \pm 73 \mu \mathrm{M}$, vanillin). Data were collected from 25-30 cells in 3 independent experiments. (C) Binary mix of eugenol (100 $\mu \mathrm{M})$ and MIEG (1 $\mathrm{mM}$ ) as well as ligands alone were applied at indicated time. (D) Another potent agonist, vanillin (100 $\mu \mathrm{M})$ was antagonized by MIEG $(1 \mathrm{mM})$ but required pre-incubation for 30-s to achieve near complete inhibition. Traces represent mean \pm SE measured in 20-30 cells. (E) Responses in all groups were normalized to the control response evoked by eugenol alone. Carvone ( $1 \mathrm{mM}, \mathrm{Carv})$ was used as a negative control imposing no antagonism in the mix with eugenol. Data represent three independent experiments. Wilcoxon paired t-test was applied, ${ }^{\star} \mathrm{p}=0.017 ;{ }^{\star \star} \mathrm{p}=0.0014 ;{ }^{\star \star \star} \mathrm{p}=0.0001$.
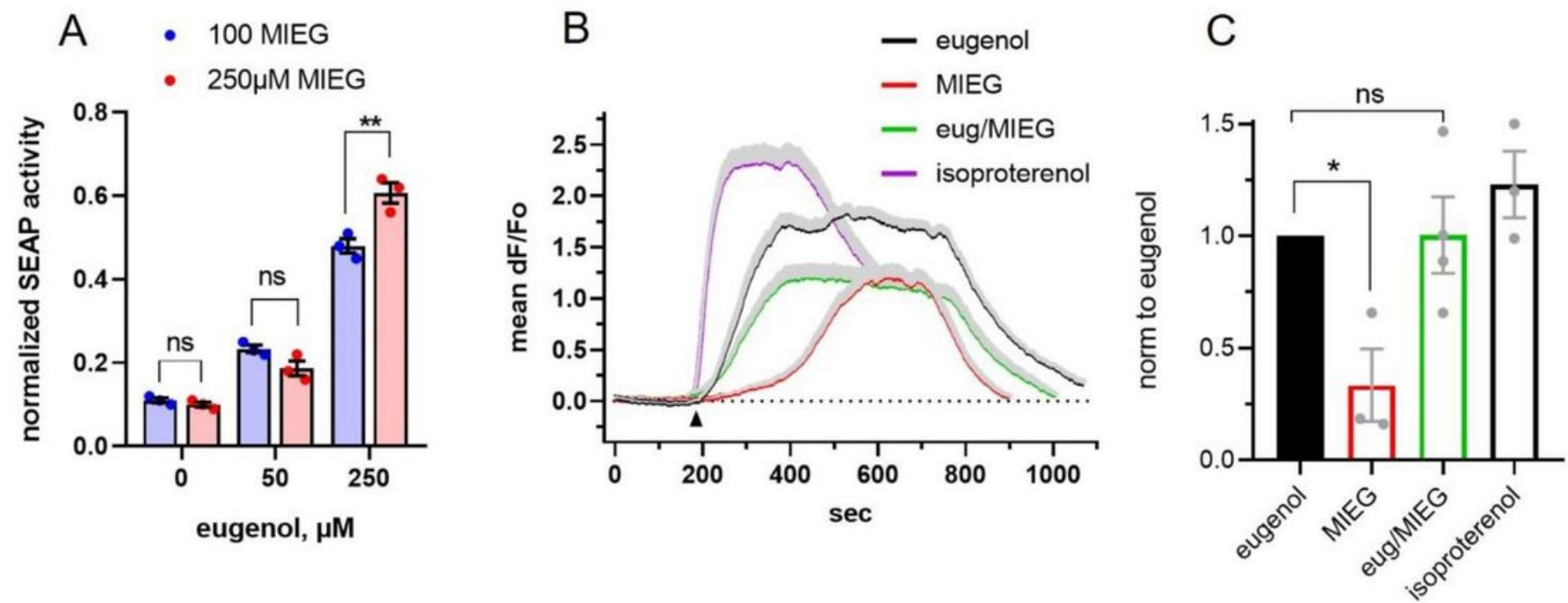

\section{Figure 6}

Prolonged exposure to Olfr73 ligands activates sustained CAMP build-up and reveals a loss of antagonism in a binary mix of eugenol and MIEG. (A) HEK293 cells co-expressing untagged Olfr73 generated cAMP following a 30-min incubation with MIEG alone or its mixture with eugenol. CAMP was measured indirectly by the activity of Cre-SEAP (multiple t-test, $p=0.288(0 \mu \mathrm{M}$ eugenol); $p=0.077(50 \mu \mathrm{M}$ eugenol); ${ }^{*} \mathrm{p}=0.013(250 \mu \mathrm{M}$ eugenol)). (B) Time-resolved cAMP-dependent activity measured by GCaMP3 in HEK293 cells co-expressing untagged Olfr73 and CNGCmut channel, a cAMP sensor. Eugenol $(100 \mu \mathrm{M})$, MIEG $(1 \mathrm{mM})$ and their binary mix were applied for $10 \mathrm{~min}$ at the time indicated (arrowhead). Isoproterenol $(10 \mu \mathrm{M})$ was applied for $5 \mathrm{~min}$ at the end of the experiment to confirm the functionality of cells and that the response to ligands was not saturating. Traces represent average (solid line) and SEM (gray shadow) measured in the same group of 102 cells. (C) Ligand induced cAMP-dependent activity was measured in at least three independent experiments at the plateau of the response and normalized to that evoked by eugenol alone. Wilcoxon paired t-test, ${ }^{*} p=0.018$ (MIEG, $\left.n=3\right) ; p=0.875$ (eug/MIEG, $n=4$ ).

\section{Supplementary Files}


This is a list of supplementary files associated with this preprint. Click to download.

- supplmovieS1ratknob.avi

- supplmovieS2mouseknob.avi

- supplmovieS3eug.avi

- supplmovieS4eugmieg.avi

- ukhanovmanuscriptSciRepfinalsupplfigures.docx 